\title{
Neurotrophic support by traumatized muscle-derived multipotent progenitor cells: Role of endothelial cells and Vascular Endothelial Growth Factor-A
}

Heidi R. H. Zupanc ${ }^{1,2}$, Peter G. Alexander ${ }^{2}$ and Rocky S. Tuan ${ }^{1,2^{*}}$

\begin{abstract}
Background: Adult mesenchymal stem cells (MSCs) have been shown to increase nerve regeneration in animal models of nerve injury. Traumatized muscle-derived multipotent progenitor cells (MPCs) share important characteristics with MSCs and are isolated from severely damaged muscle tissue following surgical debridement. Previous investigations have shown that MPCs may be induced to increase production of several neurotrophic factors, suggesting the possible utility of autologous MPCs in peripheral nerve regeneration following injury. Recent findings have also shown that components of the vascular niche, including endothelial cells (ECs) and vascular endothelial growth factor (VEGF)-A, regulate neural progenitor cells and sensory neurons.
\end{abstract}

Methods: In this study, we have investigated the neuroinductive activities of MPCs, particularly MPC-produced VEGF-A, in the context of an aligned, neuroconductive nerve guide conduit and the endothelial component of the vascular system. Embryonic dorsal root ganglia (DRG) seeded on poly- $\epsilon$-caprolactone aligned nanofibrous scaffold (NF) constructs and on tissue culture plastic, were cocultured with induced MPCs or treated with their conditioned medium (MPC-CM).

Results: Increased neurite extension was observed on both NF and tissue culture plastic in the presence of MPC-CM versus cell-free control CM. The addition of CM from ECs significantly increased the neurotrophic activity of induced MPC-CM, suggesting that MPC and EC neurotrophic activity may be synergistic. Distinctly higher VEGF-A production was seen in MPCs following neurotrophic induction versus culture under normal growth conditions. Selective removal of VEGF-A from MPC-CM reduced the observed DRG neurite extension length, indicating VEGF-A involvement in neurotrophic activity of the CM.

Conclusions: Taken together, these findings suggest the potential of MPCs to encourage nerve growth via a VEGF-A-dependent action, and the use of MPC-CM or a combination of MPC and CM from ECs for peripheral nerve repair in conjunction with NFs in a nerve guide conduit. Due to the ease of use, application of bioactive agents derived from cultured cells to enhance neurotrophic support presents a promising line of research into peripheral nerve repair.

Keywords: Blast trauma, Muscle stem cells, Mesenchymal stem cells, Peripheral nerve repair, Neurotrophic factors, Paracrine mechanism, Endothelial cells, VEGF-A

\footnotetext{
* Correspondence: rst13@pitt.edu

'Department of Bioengineering, Swanson School of Engineering, University

of Pittsburgh, Pittsburgh, PA 15219, USA

${ }^{2}$ Center for Cellular and Molecular Engineering, Department of Orthopaedic

Surgery, University of Pittsburgh School of Medicine, 450 Technology Drive,

Room 221, Pittsburgh, PA 15219, USA
} 


\section{Background}

Within a critical time frame, nerve injury healing requires the careful orchestration of several independent but related processes [1]. When a nerve injury surpasses the length of a critical-sized defect, the ideal nerve healing rate of about one inch per month [2] may be limited by fibrotic scar formation. The target tissue slowly loses the ability to recruit the recovering nerve or encourage reinnervation [3]. While several strategies have been developed to stimulate bridging of the injury gap by accelerating nerve growth, the clinical treatment of choice, grafting of autologous nerve or blood vessels, promises no guarantee of recovery and requires precise and sometimes unattainable donor tissue geometry as well as a taxing, second surgery for tissue harvest [4]. When autologous donor tissue is unattainable, patients can be treated with synthetic, biodegradable nerve guides as a last resort, but such guides are currently associated with less favorable outcomes [5].

Exciting research in recent years has attempted to increase the neurotrophic potential of these nerve guide conduits, the most sophisticated of which attempts to mimic the physical, chemical, and temporal aspects of the native repair processes. The most basic function of these guides isolates the damaged nerve, concentrating neurotrophic signals from the distal stump while eliminating unwanted, scar-forming immune cell infiltration [6]. This cellular isolation must also allow constant nutrient exchange for the sensitive nerves. These competing concerns have been addressed in recent years by tightly controlling the porosity and thickness of nerve guide conduits [6]. Within that context, substrate patterning or the incorporation of aligned fibers mimic bands of Büngner formed by proliferating, reparative Schwann cells [7, 8]. Advancements in polymer chemistry and refinements of techniques in fibrous scaffold fabrication, such as electrospinning, allow the precise control of the fiber diameter, alignment, and degradation rate $[9,10]$ with the ultimate goal of complete scaffold replacement by native tissue. The clinically approved biomaterial, poly- $\varepsilon$-caprolactone (PCL) is a biodegradable synthetic polymer that can be easily utilized for the fabrication of electrospun micro- or nanofibers [11], with a degradation rate in vivo comparable to the rate of native tissue replacement [12]. Beyond providing a permissive architecture, PCL does little to increase nerve growth. To address this relative inertness, stem cells or growth factor augmentations of these guides represent a popular topic of research, including the incorporation of bioactive, neurotrophic factors normally secreted by Schwann cells [13]. These include nerve growth factor (NGF), brain-derived neurotrophic factor (BDNF), ciliary neurotrophic factor (CNTF), and glial cell-derived neurotrophic factor (GDNF). These factors can be tethered to or encapsulated within the nerve guide conduits for controlled release [8, 14, 15].

The bioactivity of exogenously delivered factors may degrade with time, and experiments have shown that direct transplantation of biologically responsive cellular growth factor sources, such as Schwann cells and Schwann cell-like bone marrow-derived mesenchymal stem cells (MSCs), can enhance nerve repair outcomes $[16,17]$. It has been reported that nerve coculture with or treatment with conditioned medium (CM) derived from Schwann cells, Schwann-like MSCs, or undifferentiated MSCs positively influenced the length and density of neurons dissociated from chick embryonic dorsal root ganglia (DRG) [18, 19].

Ancillary to supporting the damaged nerve, MSCs are thought to signal the surrounding tissue and (possibly) invading immune cells with a resulting net positive effect on nerve defect healing $[20,21]$. The use of allogeneic MSCs, while being tested for certain clinical therapies, faces intensifying scrutiny over their immunogenicity [22]. Current sourcing of sufficient numbers of autologous MSCs also involves invasive secondary procedures. A safe and efficacious autologous MSC isolation method, integrated with standard surgical treatment methods, is thus highly desirable.

Growing evidence suggests that skeletal muscle contains multipotent stem or progenitor cells [23]. MDSCs (muscle-derived stem cells), a preplating selected cell type related to pericytes or mesenchymal stem cells, have been shown to possess multilineage differentiation abilities [24]. Recent studies have shown that careful muscle tissue and cell isolation from the viable margins of surgical waste obtained during debridement of a blast-traumatized extremity yields a population of muscle-derived multipotent progenitor cells (MPCs) [25]. As described in this article, MPCs are defined by their isolation from traumatized muscle and rapid (24 h) adherence to tissue culture plastic. MPCs should not to be confused with MDSPCs, which are derived from nontraumatized muscle and adhere slowly to collagencoated surfaces [26]. In addition to other functions, MPC neurotrophic activities and neurotrophic factor expression [27] in vivo are similar to those of widely known and clinically utilized bone marrow-derived MSCs [28], suggesting that MPCs might be an ideal candidate cell type for autologous nerve repair for victims who have suffered severe trauma and thus present readily accessible traumatized tissue specimens. To enhance the clinical utility of MPCs and the production of neurotrophic factors, MPCs can be neurotrophically induced following culture in a defined medium [27]; CM obtained following this induction can then stimulate neurite outgrowth in embryonic DRG [29]. Recent work also suggests that MPCs might convey neurotrophic benefits 
through indirect means, as MPCs exhibit the ability to control their local proteolytic microenvironment [30]. One manner of MSC tissue support might be through an association with vascular endothelial cells (ECs), both in the maintenance of a stem cell niche and in endogenous regenerative processes [31-33].

ECs are intimately connected with the development, growth, and continued health of nerves in the neurovascular unit $[17,34]$. Neural and vascular cells often utilize similar molecular pathways for physical guidance [35], which could be exploited by MSC- or MPC-based therapies. There is ample evidence that the EC ligands, vascular endothelial growth factor (VEGF)-A and angiopoietin (ANGPT)-1, also exert positive effects on neurons [36]. VEGF-A receptor (VEGFR)2 is expressed by neurons [37], and recent clinical studies have reported that VEGF-A promoted anatomical and functional recovery of injured peripheral nerves in the avascular cornea [38], but the role of VEGF-A signaling with respect to MSC- and EC-associated peripheral nerve repair remains undefined.

Extrapolating from these findings, we hypothesize that MPCs positively modulate nerve growth activities and that this effect could be augmented through interactions with ECs and physical contact guidance. These interactions were investigated using the embryonic DRG model in two experimental set-ups: (1) treatment of DRGs cultured on tissue culture plastic with one or more cellconditioned media (CMs); and (2) coculturing of DRGs and MPCs seeded onto an aligned nanofibrous biomaterial scaffold, simulating the microenvironment within a polymeric nerve conduit construct. The results reported here show that neurotrophic differentiation of MPC strongly affected DRG neurite extension on nanofibers, regardless of the presence or absence of ECs. Results from the $\mathrm{CM}$ investigations revealed that VEGF-A or VEGF-A-associated molecules in MPC-CM, derived from cultures under neurotrophic or growth conditions, were responsible for a large portion of the observed neurite length enhancement effect.

\section{Methods}

\section{Nanofiber construct formation and assessment}

Aligned nanofiber constructs (NFs) were deposited reproducibly on glass slides (Fisher, Pittsburgh, PA) by electrospinning, at $\sim 15 \mathrm{kV}$ difference from a $22-\mathrm{G}$ needle placed $15 \mathrm{~cm}$ from a rotating mandrel, an 11.5\% PCL (Sigma-Aldrich, St. Louis, MO) solution dissolved in 1:1 tetrahydrofuran:dimethylformamide (Sigma-Aldrich) [39]. NFs were prepared for cell culture by lining each edge with silicone sealant (RTV 734; Dow, Midland, MI); following an overnight cure, residual solvent was removed by storage for an additional night under vacuum with dessicant. Each slide was then disinfected with $70 \%$ ethanol (Fisher) and $10 \mathrm{~min}$ of UV-V light exposure (100-280 nm) (LEDwholesalers; Hayward, CA), Finally they were rinsed with Dulbecco's phosphate-buffered saline (PBS; Invitrogen, Carlsbad, CA). After preparation, NFs were incubated in PBS with either serial applications of $100 \mathrm{ng} /$ $\mathrm{ml}$ poly-L-lysine and $10 \mu \mathrm{g} / \mathrm{ml}$ laminin (Sigma-Aldrich) at $4{ }^{\circ} \mathrm{C}$ overnight, or with $10 \%$ fetal bovine serum (FBS; Invitrogen) at $4{ }^{\circ} \mathrm{C} 2 \times$ overnight. Fiber thickness and orientation were determined using scanning electron microscopy and plugins developed for ImageJ/Fij (BoneJ 1.4.0 [40]; Directionality 2.0 [41]).

\section{Culture of traumatized muscle-derived MPCs and ECs}

Traumatized muscle-derived MPCs, isolated as described previously [28] (four male patients, average age 24 years), were expanded from low initial cell numbers to passage 5-8 in tissue culture-treated flasks (Fisher, Nunc, Rochester, NY) in growth medium (GM): $\alpha$-minimum essential medium (MEM) $+10 \% \mathrm{FBS}+$ penicillin-streptomycin-fungizone (PSF) $+1 \mathrm{ng} / \mathrm{ml}$ basic fibroblast growth factor (FGF)-2 (all reagents from Invitrogen). Human dermal microvascular ECs (HUVECs) were obtained from the Centers for Disease Control and Prevention (via Material Transfer Agreement) [33] and cultured on tissue culturetreated flasks in EGM-2MV medium (Lonza, Basel, Switzerland). At 90\% confluency, cells were trypsinized (Invitrogen) and passaged at $1 \times 10^{6}$ cells/T150 flask. All cells were cultured at $37^{\circ} \mathrm{C}$ under $5 \% \mathrm{CO}_{2}$.

Twenty-four hours after passage, $\mathrm{CM}$ was generated by culturing cells (denoted GM-MPC or EC) for $48 \mathrm{~h}$ in basal medium, containing Dulbecco's modified Eagle's medium $(\mathrm{DMEM})+1 \%$ insulin-transferrin-selenium-ethanolamine (ITS-X; Life Technologies, Carlsbad, CA) + penicillinstreptomycin (PS; Invitrogen). CM was denoted MPC-CM, EC-CM, or acellular control when derived from MPCs, ECs, or cell-free basal medium incubated with the same substrate, respectively. Following harvest, CM was prepared by centrifugation at $200 \mathrm{~g}$, stored frozen at $-80{ }^{\circ} \mathrm{C}$ until use, and thawed and centrifuged again just prior to use.

\section{Neurotrophic induction}

MPCs were seeded at $1 \times 10^{3}$ cells $/ \mathrm{cm}^{2}$ on tissue culture plastic or NFs and differentiated neurotrophically using a modified 10-day protocol [27, 29, 42]. Twenty-four hours after seeding, MPCs were incubated with GM supplemented with $10 \mathrm{mM} \beta$-mercaptoethanol (Sigma) for $24 \mathrm{~h}$, followed by $48 \mathrm{~h}$ in GM supplemented with $10 \mathrm{mM} \quad \beta$-mercaptoethanol $+35 \mathrm{ng} / \mathrm{ml}$ retinoic acid (Sigma). For the 6 following days, MPCs were incubated with neurotrophic medium (DMEM/Ham's F12 + PSF (Invitrogen) supplemented with 2\% FBS, 2\% B-27 (Invitrogen), $6 \mathrm{mg} / \mathrm{ml}$ retinoic acid, $1 \mathrm{ng} / \mathrm{ml} \mathrm{FGF-2} \mathrm{(Sigma),}$ $10 \mathrm{ng} / \mathrm{ml}$ platelet-derived growth factor (PDGF; Sigma), 
$150 \mathrm{ng} / \mathrm{ml}$ heregulin (an isoform of neuregulin-1; Sigma), and $10 \mu \mathrm{M}$ forskolin (Sigma)).

Following neurotrophic differentiation, MPCs (designated nMPCs) were washed with PBS and either lysed with TRiZol, or incubated with basal medium (see above) for $48 \mathrm{~h}$ to produce conditioned medium (nMPC-CM). For DRG coculture experiments, tissue culture plastic-cultured nMPCs were trypsinized and transferred to DRG-containing fibers at a concentration of $1 \times 10^{3} \mathrm{cells} / \mathrm{cm}^{2}$.

\section{EC/MPC culture on nanofibrous constructs}

MPCs and ECs were seeded on FBS-coated NFs at various densities $\left(\right.$ cells $\left./ \mathrm{cm}^{2}\right)$ in cell-specific growth medium; medium was exchanged every 48 h. Cell viability was assessed by quantifying the number of fluorescent live (green) and dead (red) cells visible within each microscopy image after Live/Dead staining (Invitrogen). Fluorescent cells were visualized with an Olympus inverted microscope equipped with a motorized stage controlled through MetaMorph. Whole cell numbers were determined automatically for each image by setting thresholds for fluorescence intensity, cell size, and cell shape using custom macros written for Image//Fiji [43]. Cell density was determined by scaling the number of live cells observed in each image to $1 \mathrm{~cm}^{2}$.

\section{Nerve DRG culture and confirmation of MPC/EC neurotrophic activity}

DRGs were obtained via microdissection from incubation day 9 chicken embryos, and placed on poly-L-lysine- and laminin-coated (Sigma) tissue culture plastic [44, 45]. After $24 \mathrm{~h}$ in nerve growth medium (basic Eagle's medium, 10\% horse serum, $1 \mathrm{nM}$ GlutaMax; Invitrogen) supplemented with NGF + epidermal growth factor (EGF) + PDGF, DRGs were cultured for 4 additional days in a combination medium of 1:1 CM:DRG basal medium. CM-supplemented media were exchanged daily.

To assess the effect of MPC or EC coculture on DRG neurite extension, freshly isolated DRGs were seeded on sterile, aligned poly-L-lysine- and laminin-coated NFs. After $24 \mathrm{~h}$ in nerve growth medium, trypsinized MPCs, nMPCs, ECs, or a 1:1 xMPC-EC mixture were seeded onto DRG-containing NFs at a final density of $1 \times 10^{4}$ cells $/ \mathrm{cm}^{2}\left(1 \times 10^{5}\right.$ per NF) in $1 \mathrm{ml}$ of DRG basal medium. Control cultures were incubated in only $1 \mathrm{ml}$ of DRG basal medium. All media were exchanged daily for 4 additional days, and DRG neurite outgrowth was assessed as described previously.

\section{Selective VEGF-A depletion}

VEGF-A was selectively removed from thawed, centrifuged, and filtered $\mathrm{CM}$ using neutralizing antibodies (mouse IgG, mouse aVEGF-A; R\&D, Minneapolis, MN) bound to SpinTrap Protein G beads (Sigma). Beads and bound antibodies/factors were removed from $\mathrm{CM}$ by centrifugation at $200 \mathrm{~g}$ for $5 \mathrm{~min}$. Bound VEGF-A was eluted from the beads, and selective binding was confirmed via VEGF-A enzyme-linked immunosorbent assay (ELISA; R\&D). VEGF-A-depleted or immunoglobulin (Ig)G-control CM were indicated with " $+\alpha$ VEGF-A" or “+IgG," respectively.

\section{Confirmation of neurotrophic induction}

Neurotrophic factor production/secretion into CM was assessed via sandwich ELISAs (R\&D). RNA was isolated with TRiZol, reverse transcribed, and analyzed by reverse-transcription polymerase chain reaction (RTPCR) for neural gene expression (Superscript III (Invitrogen) 18S rRNA, BDNF, CNTF, GDNF, Nestin, NGF, VEGF-A (Qiagen, Hilden, Germany)).

\section{Neurite outgrowth measurement}

To assess the effect of CM or MPC/EC coculture on neurite outgrowth, DRGs were fixed and stained immunohistochemically for heavy neurofilament (NEFH; Sigma) (Secondary Alexa-Fluor antibodies; Invitrogen). DRGs were visualized with an Olympus inverted microscope equipped with a motorized stage controlled through MetaMorph. Resultant mosaic images were stitched using Grid/Collection Stitching (Fiji) [46]. Each image represented one replicate. The 10 longest NEFH-positive neurite extensions were measured from the geometric center of the original DRG cluster (ImageJ).

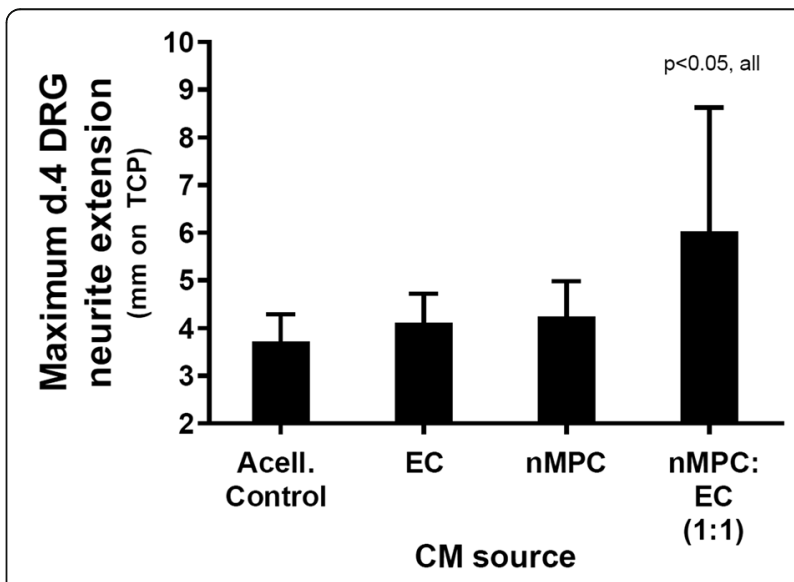

Fig. 1 Neurite extension of dorsal root ganglia (DRGs) seeded on tissue culture plastic and cultured in the presence of conditioned medium (CM) from multipotent progenitor cells (MPCs) and/or endothelial cells (ECs). An increase in DRG neurite extension was detected as a synergistic effect of the mixed (1:1) CM derived from neurotrophically-induced MPCs (nMPC) and EC over the basal DRG media acellular control (Acell. control). $n=6$; Tukey's. Indicated $p$ value applies to that condition versus all other conditions 


\section{Statistical analysis}

All data are presented as mean \pm standard deviation, unless noted. Statistical differences and $p$ values were determined by one- or two-way analysis of variance (ANOVA) and Sidak's or Tukey's test, as appropriate.

\section{Results}

Neurotrophic support by MPCs and ECs cultured on tissue culture plastic

To assess the relative trophic properties of the different cell types, conditioned medium (CM) from the various cell types or basal (control) medium was incubated with tissue culture plastic-seeded DRGs. In the presence of CM derived from ECs or neurotrophically induced MPCs, DRG neurite extensions increased slightly (but not significantly) above control lengths (Fig. 1). By contrast, DRG neurite extension lengths increased to almost twice that of the basal medium control in the presence of a combination of the $\mathrm{CM}$ from both cell types. This finding suggested that a combination of MPC and EC neurotrophic activities might better support neurite extension on a nerve guide conduit than either cell type in isolation.

\section{Nanofiber conduit (NF) fabrication}

Efficient nanofiber-based physical guidance of neurite outgrowth requires the presence of appropriately sized (nanoscale) parallel fibers. Because batch-to-batch consistency of electrospun fibers is notoriously low [47], randomly selected scaffolds from multiple batches of NFs were examined using scanning electron microscopy. Fiber diameter was quantified through image analysis and suggested fairly consistent nanofiber diameter (580 $\pm 280 \mathrm{~nm})$ and relatively good alignment $\left(22 \pm 17^{\circ}\right.$ dispersion).

\section{Cell viability of effector cells on NFs}

To ensure that DRG-effector cell cocultures included sufficient space and nutrients for all cell types, including oxidative stress-sensitive nerve cultures [48, 49], effector cells were seeded on NFs and assessed for their longterm $(>24 \mathrm{~h})$ viability and density $\left(\right.$ cells $\left./ \mathrm{cm}^{2}\right)$. MPCs or
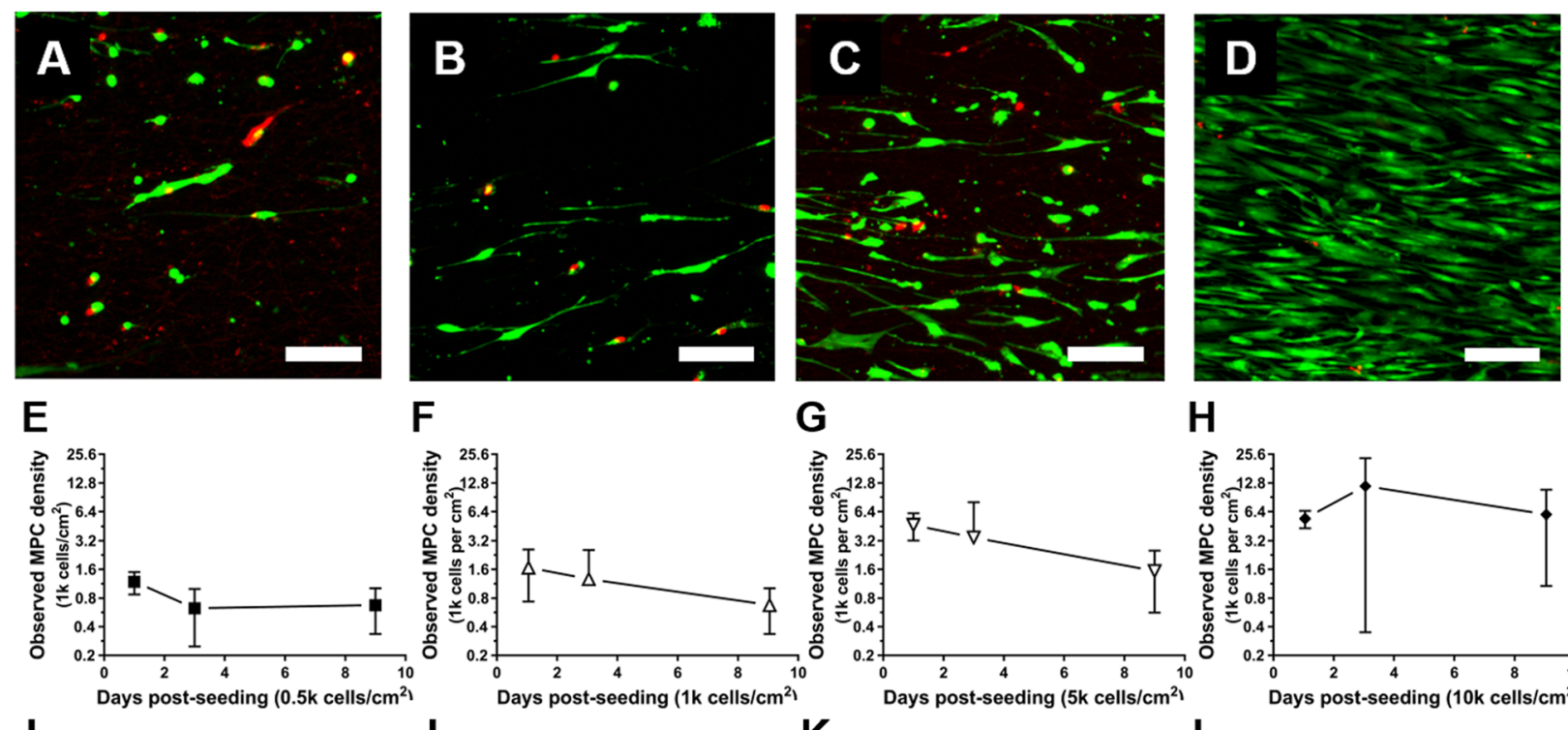

$\mathrm{H}$
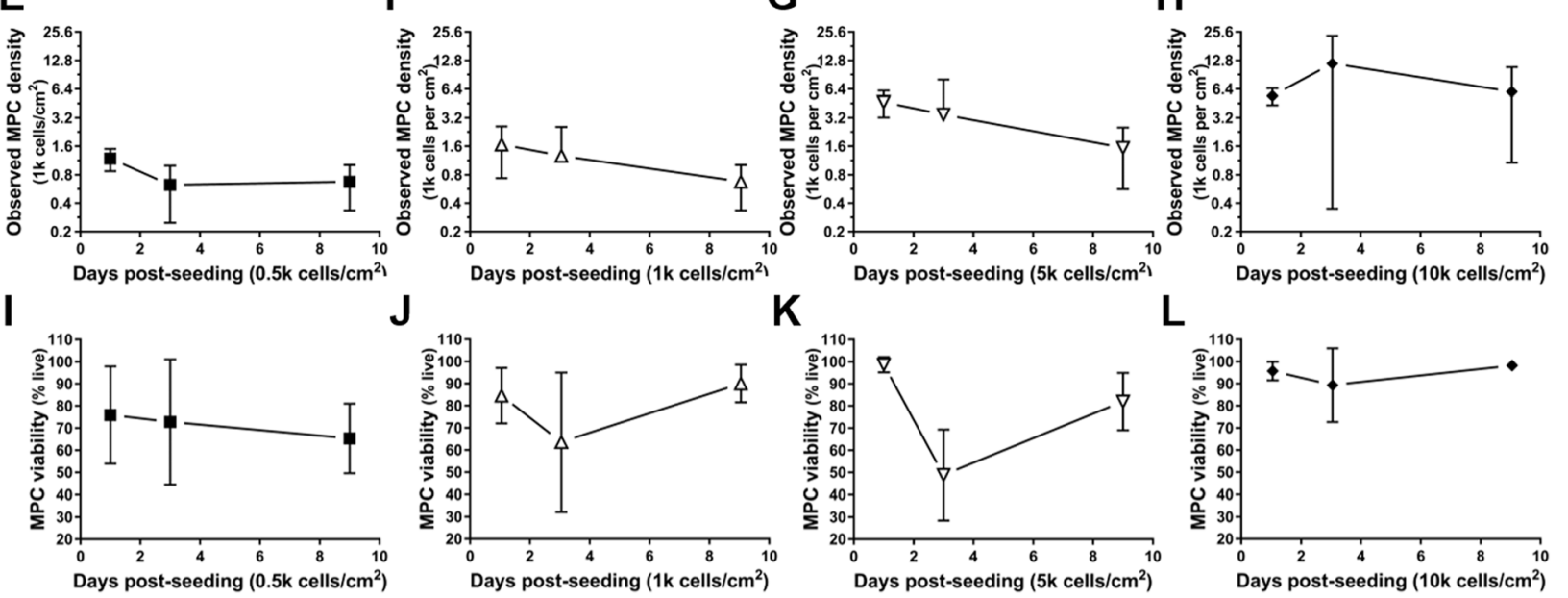

Fig. 2 Viability and density of multipotent progenitor cells (MPCs) seeded on aligned NF scaffold. Edge-to-edge mosaic images of NF-seeded MPCs stained with Live/Dead (green/red) assay were obtained at 24 h post-seeding at (a,e,i) $0.5,(\mathbf{b}, \mathbf{f}, \mathbf{j}) 1,(\mathbf{c}, \mathbf{g}, \mathbf{k}) 5$, and $(\mathbf{d}, \mathbf{h}, \mathbf{l}) 10 \times 10^{3}$ (k) cells/cm²; scale bar $=100 \mu \mathrm{m}$. Evidence of NF-mediated cell alignment could be seen in the common orientation of the MPCs. e-h MPC density (presented as an exponential scale) as a function of culture time. MPC density scaled with initial seeding density at early time points (days 1 and 3), but eventually converged towards $1 \mathrm{k}$ cells $/ \mathrm{cm}^{2}$ by day 9 , except for the $10 \mathrm{k}$ cultures. Cell aggregation on the scaffolds resulted in extreme local variations in cell density. MPC viability (i-I) varied widely at early time points. By day $9,65 \%$ or more cells remained viable, and viability increased with initial seeding density 
ECs were initially seeded on $10 \mathrm{~cm}^{2}$ serum-coated NFs at varying densities $\left(0.5,1,5,10 \times 10^{3}\right.$ cells $\left./ \mathrm{cm}^{2}\right)$. Live cell density and percent viability were assessed daily using metabolic stain (Live-Dead stain) for the first 3 days and after an additional week in culture (Fig. 2), corresponding to the schedule of neurotrophic induction. Cells were cultured in their respective growth media to allow for maximum proliferation.

MPCs tolerated NF culture well, reaching an equilibrium density of approximately $1-5 \times 10^{3}$ cells $/ \mathrm{cm}^{2}$, but EC coverage and viability dropped steadily over the culture period. Cell aggregation contributed to large variations in within- and between-sample viability and density measurements.

Because of these findings, subsequent experiments involving NF scaffolds were examined after short culture ( $<5$ day) and at low-moderate initial cell densities $(1 \times$ $10^{3} / \mathrm{cm}^{2}$ ). To examine longer term ( $>5$ days) effects on neurite extension and neurotrophic differentiation, ECs and MPCs were cultured on tissue culture plastic.

\section{Influence of effector cell coculture on DRG neurite extension}

To assess the potential neurotrophic effect of the combination of MPCs/nMPCs and ECs (effector cells) in the context of a nerve guide, DRGs and effector cells were seeded onto NFs and cocultured. Prepared NFs were first seeded with DRGs. Twenty-four hours after DRG seeding on the NFs, cocultures were constructed by adding effector cells. Effector cells consisted of ECs, noninduced MPCs, nMPCs, and 1:1 EC-xMPC mix (co-effectors). Effector cells were added to the NFs at a final effector cell density of $1 \times 10^{3}$ cells $/ \mathrm{cm}^{2}$. NEFHpositive DRG neurite extensions were assessed after 4 days of coculture with daily medium changes (Fig. 3).

In the presence of MPC effector cells and regardless of the MPC neurotrophic induction, DRG neurite extensions increased. Specifically, DRG neurite lengths in nMPC-DRG cocultures were 3- to 4-fold longer than DRG neurite lengths observed in control cultures (NFseeded DRGs with no effector cells). This finding confirmed the functional utility of the neurotrophic differentiation protocol. The additional presence of ECs on the NFs caused a trend towards an increase in DRG neurite length with the longest extensions observed under co-effector coculture conditions. This effect suggests that a nerve guide conduit could promote nerve growth by incorporating a combination of MPCs and ECs.

\section{NF effects on effector cell neurotrophic activities}

To assess whether NF seeding might have altered MPC neurotrophic functions, MPCs were differentiated on NF
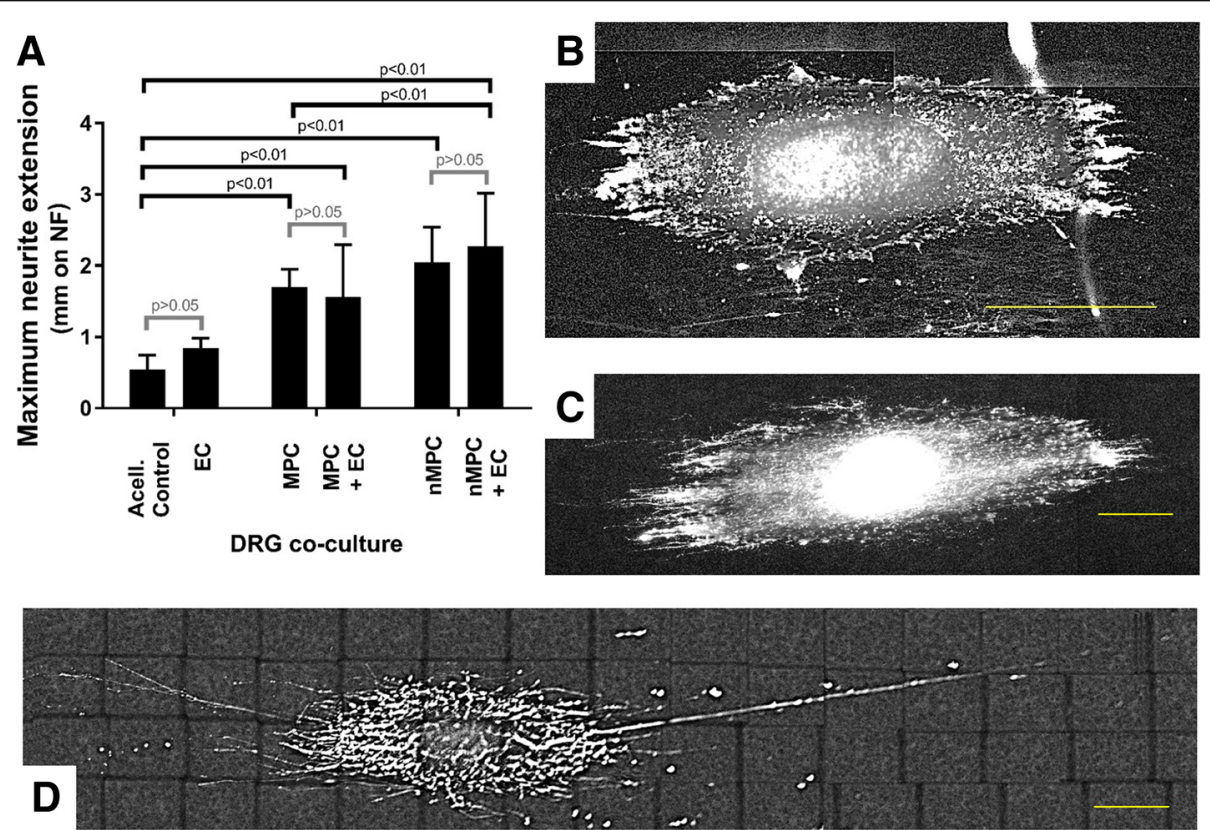

Fig. 3 Neurite extensions of dorsal root ganglia (DRGs) seeded on nanofiber NF scaffold upon coculture with multipotent progenitor cells (MPCS) or combination of MPCs/endothelial cells (ECS) (1:1). The presence of MPCs, particularly after their neurotrophic induction, increased the length of DRG neurite extensions (a) over DRG control (acellular (Acell.) control/basal medium) cultures (b). EC coculture (c) slightly but not significantly increased DRG neurite extension length. MPC and neurotrophically-induced MPC (nMPC) coculture significantly increased neurite extension length when compared to the control. For XMPC-DRG coculture, EC coculture slightly but insignificantly increased neurite extension length. DRGs cocultured with both nMPC and EC (nMPC-EC-DRG cocultures; $\mathbf{d}$ ) exhibited significantly longer neurite extensions than MPC-DRG or MPC-EC-DRG cocultures. a $n=4$; Sidak's $p$ values as indicated. $\mathbf{b}-\mathbf{d}$ Scale bars $=400 \mu \mathrm{m}$ for representative NEFH-stained images 
and tissue culture plastic by seeding cells in induction medium onto each surface at densities of $1 \times 10^{3}$ cells $/ \mathrm{cm}^{2}$. Except for an increase in VEGF-A expression, neurotrophic gene expression of NF-seeded nMPCs did not change significantly compared to tissue culture plastic-seeded nMPCs (Fig. 4a and b).

However, pooled CM from NF-seeded nMPCs exhibited much lower factor concentrations than $\mathrm{CM}$ from similarly induced tissue culture plastic-seeded nMPCs. $\mathrm{CM}$ derived from tissue culture plastic-cultured nMPCs contained FGF-2 $(130 \pm 220 \mathrm{pg} / \mathrm{ml}, n=6)$ and GDNF $(30 \pm 50 \mathrm{pg} / \mathrm{ml})$ inconsistently, with multiple samples yielding undetectable amounts of each factor. Similar to BDNF, FGF-2 and GDNF could not be detected in CM derived from NF-cultured nMPCs, and secreted CNTF and NGF could not be detected in any samples.

When compared to culture on tissue culture plastic, EC culture on NF resulted in significant changes in EC neurotrophic gene expression (Fig. 4c and d). Despite these gene expression changes, only BDNF and VEGF-A could be detected in EC-CM from either substrate (tissue culture plastic or NF). NF culture severely reduced the level of neurotrophic growth factors measured in EC-CM. This observation and the above note of diminished cytokine and growth factor concentrations in NFseeded cultures suggests that protein-scaffold interactions may restrict cytokine and growth factor release [50]. This likely explains the diminished relative neurite extension observed upon MPC or EC coculture with
DRGs (Fig. 3) versus tissue culture plastic-derived CM neurotrophic support (Fig. 1).

\section{Mechanism of action of ECs and MPCs}

Interestingly, our analysis showed that VEGF-A, most commonly known for its role in angiogenesis and known to be expressed robustly by MPCs, was secreted by MPCs in copious amounts upon neurotrophic induction (Fig. 4b and d). The magnitude of secreted VEGF-A relative to other neurotrophic factors as well as the observation of VEGFR [51] on the surface of nerves suggested that it might play a more significant role in MPCmediated neurotrophic activity than previously realized. To test whether VEGF-A was acting as a mediator of the MPC effect on DRG, VEGF-A was selectively depleted from nMPC-CM, EC-CM, and VEGF-A-spiked medium using anti-VEGF-A antibodies bound to Protein $G$ beads. Effective VEGF-A immunodepletion was confirmed by ELISA (Fig. 5i). VEGF-A concentrations in control and IgG-treated medium were identical $(\sim 170 \mathrm{pg} / \mathrm{ml})$, and VEGF-A concentrations in VEGF-A-depleted samples were below the detection limit. VEGF-A removal from CM derived from nMPCs (Fig. 5d) and ECs (Fig. 5f) significantly decreased the maximum observed length of DRG neurite extensions when cultured on tissue culture plastic (Fig. 5a-g), suggesting a dependence of the neurotrophic activity of both cell types on VEGF-A in addition to other MPC-secreted neurotrophic compounds.
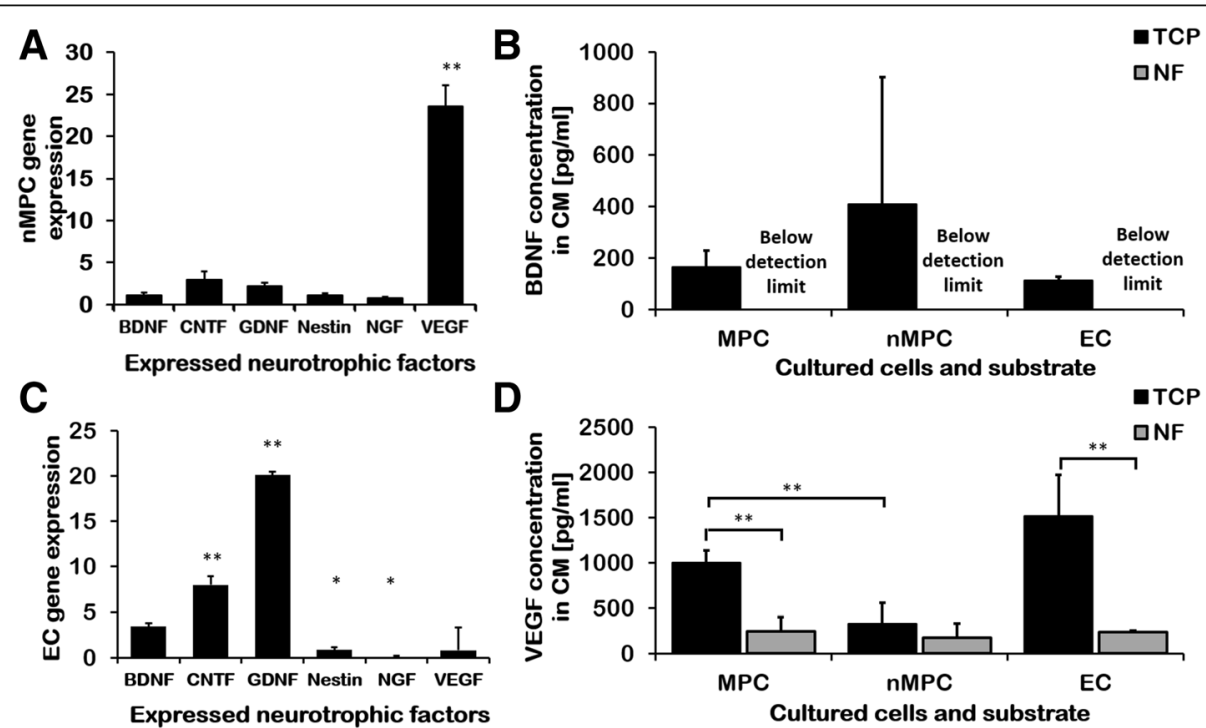

Fig. 4 Expression and production of neurotrophically induced MPCs (nMPC) and endothelial cells (EC) cultured on tissue culture plastic (TCP) and nanofiber constructs (NF). a,c NF-cultured growth factor and neurotrophic marker gene expression, assayed by RT-PCR and normalized to TCP cultures: secreted brain-derived neurotrophic factor (BDNF) (b) or vascular endothelial growth factor (VEGF) (d) levels from cells seeded on TCP or NF as measured by ELISA. NMPC (a) and EC (c) neurotrophic gene expression was mildly or significantly increased by NF culture in almost all cases. CM from cultures on NF contained lower levels of secreted growth factors than conditioned medium (CM) from TCP-cultured cells (b,d). Noninduced multipotent progenitor cells (MPCs) secreted more VEGF than nMPCs (b,d). $n=6$; Student's $t$ test ${ }^{*} p<0.05$, ** $p<0.01$, versus controls $(\mathbf{a}, \mathbf{c})$ or indicated groups $(\mathbf{b}, \mathbf{d})$. CNTF ciliary neurotrophic factor, GNDF glial cell-derived neurotrophic factor, NGF nerve growth factor 

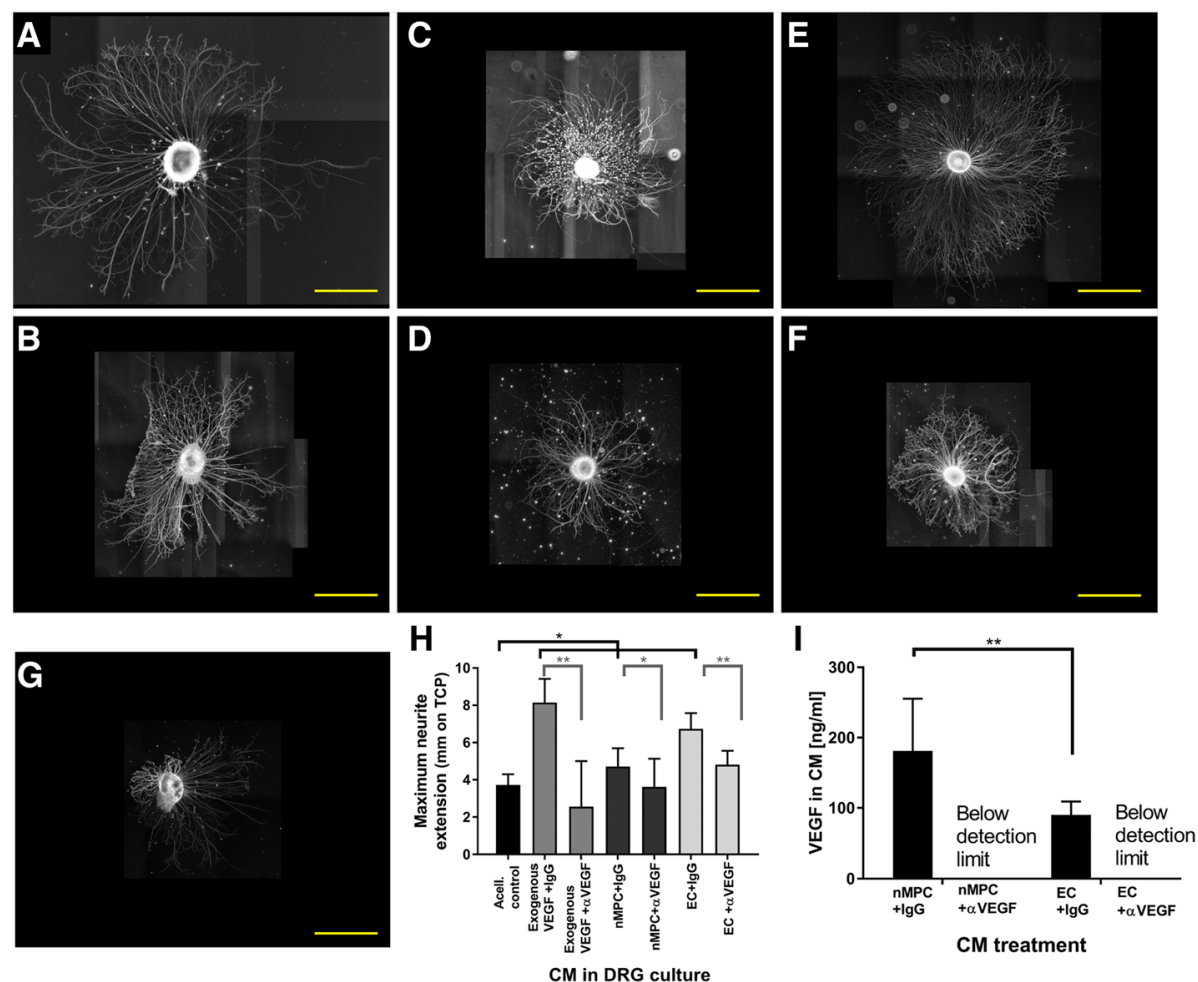

Fig. 5 Vascular endothelial growth factor (VEGF)-A production by neurotrophically induced MPCs ( $M P P C$ ) and endothelial cells (EC) positively affects neurite extension. a,c,e Dorsal root ganglia (DRG) neurite extensions in the presence of conditioned medium (CM) and immunoglobulin $\mathrm{G}$ (IgG) control antibody. b,d,f DRG neurite extensions in the presence of CM and a-VEGF-A antibodies (VEGF-A immunodepletion). Compared to nonspecific IgG control (a), selective removal of VEGF-A from a positive control consisting of exogenous VEGF-A (b) resulted in the loss of neurite growth induction, validating the effectiveness of VEGF-A immunodepletion. VEGF-A immunodepletion of CM derived from nMPC (d) and EC ( $\mathbf{f}$ ) decreased observed neurite extensions compared to lgG-treated mixtures (c and $\mathbf{e}$, respectively), indicating that VEGF-A is an active component that contributes to both MPC and EC neurotrophic activities. $\mathbf{h}$ Quantification of neurite growth following depletion of VEGF-A. VEGF-A immunodepletion was associated with a significant decrease in CM-induced neurite outgrowth (one-way ANOVA, Tukey's). i VEGF-A levels in CM measured by ELISA. VEGF-A was present in CM derived from both MPCs and ECs and could be removed by immunoprecipitation using VEGF-A-specific antibodies (versus control IgG antibodies). a-g Representative NEFH-stained images; scale bar $=1 \mathrm{~mm}$. h,i $n=3$; one-way ANOVA Tukey's ${ }^{*} p<0.05,{ }^{* *} p<0.01$

\section{Discussion}

We have previously reported that neurotrophic induction of MPCs enhances their production of neurotrophic factors, including BDNF and CNTF. Extending that work, results from this study show that nMPCs could support increased DRG neurite extension on nanofiber conduits with approved nerve guide materials. This support was partially mediated through soluble factors, including those factors found in conditioned medium and secreted during MPC-DRG coculture. This support was observed regardless of the substrate on which the DRGs were seeded (tissue culture plastic or nanofiber scaffolds (NFs)), despite adverse effects of the NFs on MPC viability and measurable growth factors in the CM. Due to high betweenpatient and between-experiment variability, consistent and significant differences in VEGF-A or BDNF concentrations in the $\mathrm{CM}$ were not observed. When compared to MPCs, nMPCs were associated with distinctly higher VEGF-A concentrations as well as consistent BDNF production, similar to results reported in other work [16].
Our findings show that the combination of nMPCs and ECs preferentially encourages neurite elongation (Fig. 1). Signaling molecules from each cell type work almost synergistically despite similar levels of known neurotrophic factors, BDNF and VEGF-A, in the respective CMs (Fig. 4b and d). This enhanced neurotrophic activity could be explained in part by a mixed population of cells in the isolated MPCs. A CD29 ${ }^{+} / \mathrm{CD} 34^{+}$vascular endothelial progenitor cell (EPC)-like population has been noted within muscle isolates [52]; muscle-derived stem cells have additionally been observed to spontaneously express both vascular and neurotrophic markers upon culture with neurons [53], suggesting plasticity, cell fusion, or a culture of mixed cell types $[53,54]$.

For this investigation, nMPCs were investigated as local sources of neurotrophic factors in the context of an implanted NF. In keeping with previous studies, nMPC-CM was utilized without concentration [29]. Other researchers have reported greatly enhanced MSC trophic activities 
upon culture with cellulose fiber-concentrated CM [55], and it is possible that concentrating CM in future studies may yield stronger neural support.

To promote better DRG attachment and growth on NF substrate, laminin and lysine coatings were utilized in line with a growing consensus that many aligned fibrous structures bearing extracellular matrix (ECM) molecules are beneficial to the ultimate function of a nerve guide conduit [54]. Several groups have also investigated physical modifications to the PCL surfaces or chemical/ligand conjugation $[12,56]$ to better support initial nerve attachment and proliferation, and work is ongoing with coatings derived from various ECM molecules to increase MPC scaffold-based neurotrophic activity. Despite PCL NF culture of MPCs being unfavorable with respect to neurotrophic induction (Fig. 2), constructseeded MPCs both survived on the NFs (Fig. 2) and encouraged increases in DRG neurite extension. Furthermore, the combination coculture of ECs and nMPCs encouraged the longest neurite extensions (Fig. 3). In recent work with adipose-derived MSCs implanted in rats, Kingham et al. showed that the neurotrophic differentiation of those cells simultaneously promoted both EC vascular and DRG nerve function [57]. Taken together, these findings suggest that by optimizing the interactions of MPCs and ECs preferential neural tissue growth could be achieved. Proteomic analysis of the secretomes of both cell types might elucidate synergistic neurotrophic interactions or potential antagonistic interactions between inhibitory factors in the CM of either the MPCs or ECs, the details of which warrant further study.

Neurotrophic and angiogenic factors have been found at widely varying levels within MSC- and muscle-derived stem cell secretomes, depending on the donor and tissue source $[58,59]$. We therefore examined the potential involvement of VEGF-A in the neurotrophic potential of $\mathrm{CM}$ from ECs and nMPCs. Following immunodepletion of VEGF-A from CM, neurite extension length decreased when compared to the use of control antibodies, suggesting that VEGF-A is required for $\mathrm{EC}$ and $\mathrm{nMPC}$ promotion of neurite outgrowth (Fig. 5). This finding is consistent with other reports on the positive effect of VEGF-A on DRG neurite extension, and this work reports the first analysis of potential mechanisms for MPC enhancement of neurite extension.

\section{Conclusion}

Taken together, these findings demonstrate that the neurotrophic activities of MPCs are enhanced by biological induction in vitro, CM combination with ECs, or coculture with adult ECs. VEGF-A played a role in the observed positive effect of MPC-CM on DRG neurite extension. Given its dual role as an angiogenic and neurotrophic factor, coupled with the observation of enhanced neurite extension under EC influence, VEGFA should be incorporated in future NF-based cultures in an attempt to support both cell types. The unfavorable effect of the fibrous scaffold on MPC gene expression and measured protein secretion indicates that $\mathrm{PCL}$, without appropriate ECM coating, is likely a suboptimal substrate for nMPCs. Testing of more cell-friendly substrates as well as coating the existing PCL scaffold structure with various ECM molecules are both ongoing and should yield useful information for the development of a regenerative construct for nerve repair.

\section{Abbreviations}

BDNF: Brain-derived neurotrophic factor; CM: Conditioned medium; CNTF: Ciliary neurotrophic factor; DMEM: Dulbecco's modified Eagle's medium; DRG: Dorsal root ganglion; EC: Endothelial cell; ECM: Extracellular matrix; EGF: Epidermal growth factor; ELISA: Enzyme-linked immunosorbent assay; FBS: Fetal bovine serum; FGF: Fibroblast growth factor; GDNF: Glial cell-derived neurotrophic factor; GM: Growth medium; Ig: Immunoglobulin; MPC: Multipotent progenitor cell; MSC: Mesenchymal stem cell; NEFH: Heavy neurofilament; NF: Nanofiber scaffold; NGF: Nerve growth factor;

nMPC: Neurotrophically induced multipotent progenitor cell; PBS: Phosphate-

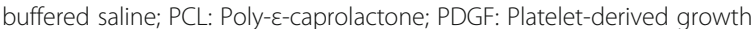
factor; PSF: Penicillin-streptomycin-fungizone; VEGF: Vascular endothelial growth factor; VEGFR: Vascular endothelial growth factor receptor; XMPC: MPC or $\mathrm{nMPC}$

\section{Acknowledgements}

This work would not have been possible without the help, advice, support, and/or guidance of the following individuals (listed alphabetically): Natasha Baker, Allison Bean, Donna Beer Stolz, Rachel Brick, Karen L. Clark, Thais Cuperman-Pohl, Solvig Diederichs, Jonathan Franks, Riccardo Gottardi, Thomas P. Lozito, Jian Tan, and Simon Watkins.

\section{Funding}

Funding support is derived in part from the Commonwealth of Pennsylvania Department of Health and US Department of Defense (W81XWH-10-2-0084, W81XWH-15-1-0600). HRHZ was a predoctoral trainee on a NIH T32 EB001026 (CATER) Training Grant.

\section{Availability of data and materials}

The datasets used and/or analyzed during the current study are available from the corresponding author upon reasonable request.

\section{Authors' contributions}

RST and PGA obtained the funding for this work, designed a portion of the experiment, advised about data analysis and reporting, and edited the manuscript for content and clarity. HRHZ designed a portion of the experiments, performed the experiments, analyzed and reported the data, wrote the manuscript, and edited the manuscript for content and clarity. A portion of this work was presented in the Ph.D. dissertation of $\mathrm{HRHZ}$ (as HRH). All authors read and approved the final manuscript.

\section{Ethics approval and consent to participate}

MPCs were derived from debrided muscle waste during surgery on blasttraumatized individuals, with patient consent, at the Walter Reed Army Medical Center, according to an IRB-approved protocol (06-24046(2)), and used in this study with approved inter-institutional Material Transfer Agreement and according to an IRB approved, exempted protocol (PRO10090075). Although IACUC approval is not required for the use of avian embryos, research with DRGs from avian embryos was performed in a manner that minimizes the suffering or discomfort of the avian embryo.

Consent for publication

Not applicable. 


\section{Competing interests}

RST is an Editor-in-Chief of the journal. The authors declare that they have no competing interests.

\section{Publisher's Note}

Springer Nature remains neutral with regard to jurisdictional claims in published maps and institutional affiliations.

\section{Received: 27 April 2017 Revised: 4 September 2017} Accepted: 8 September 2017 Published online: 13 October 2017

\section{References}

1. Belkas JS, Shoichet MS, Midha R. Peripheral nerve regeneration through guidance tubes. Neurol Res. 2004;26:151-60. doi:10.1179/ 016164104225013798.

2. Brushart T. Nerve Repair. 1st ed. New York: Oxford University Press; 2011. doi:10.1093/med/9780195169904.001.0001

3. Fu SY, Gordon T. Contributing factors to poor functional recovery after delayed nerve repair: prolonged denervation. J Neurosci. 1995;15:3886-95.

4. Deumens R, Bozkurt A, Meek MF, Marcus MA, Joosten EA, Weis J, et al. Repairing injured peripheral nerves: bridging the gap. Prog Neurobiol. 2010; 92:245-76. doi:10.1016/j.pneurobio.2010.10.002.

5. Griffin JW, Hogan MV, Chhabra AB, Deal DN. Peripheral nerve repair and reconstruction. J Bone Jt Surg Am. 2013;95:2144-51. doi:10.2106/jbjs.l.00704.

6. Kehoe S, Zhang XF, Boyd D. FDA approved guidance conduits and wraps for peripheral nerve injury: a review of materials and efficacy. Injury. 2012;43: 553-72. doi:10.1016/j.injury.2010.12.030.

7. Norman J, Desai T. Methods for fabrication of nanoscale topography for tissue engineering scaffolds. Ann Biomed Eng. 2006;34:89-101. doi:10.1007/ s10439-005-9005-4.

8. Kim Y, Haftel VK, Kumar S, Bellamkonda RV. The role of aligned polymer fiber-based constructs in the bridging of long peripheral nerve gaps. Biomaterials. 2008;29:3117-27. doi: 10.1016/j.biomaterials.2008.03.042.

9. Yang F, Murugan R, Wang S, Ramakrishna S. Electrospinning of nano/micro scale poly(L-lactic acid) aligned fibers and their potential in neural tissue engineering. Biomaterials. 2005;26:2603-10. doi:10.1016/j.biomaterials.2004. 06.051.

10. Li WJ, Mauck RL, Cooper JA, Yuan X, Tuan RS. Engineering controllable anisotropy in electrospun biodegradable nanofibrous scaffolds for musculoskeletal tissue engineering. J Biomech. 2007;40:1686-93. doi:10. 1016/j.jbiomech.2006.09.004.

11. Daly W, Yao L, Zeugolis D, Windebank A, Pandit A. A biomaterials approach to peripheral nerve regeneration: bridging the peripheral nerve gap and enhancing functional recovery. J R Soc Interface. 2012;9:202-21. doi:10.1098/ rsif.2011.0438.

12. Barbarisi M, Marino G, Armenia E, Vincenzo Q, Rosso F, Porcelli M, et al. Use of polycaprolactone $(\mathrm{PCL})$ as scaffolds for the regeneration of nerve tissue. J Biomed Mater Res A. 2014; doi:10.1002/jbm.a.35318

13. Allodi I, Udina E, Navarro X. Specificity of peripheral nerve regeneration: interactions at the axon level. Prog Neurobiol. 2012;98:16-37. doi:10.1016/j. pneurobio.2012.05.005

14. Kingham PJ, Terenghi G. Bioengineered nerve regeneration and muscle reinnervation. J Anat. 2006;209:511-26. doi:10.1111/j.1469-7580.2006.00623.x.

15. Kokai LE, Ghaznavi AM, Marra KG. Incorporation of double-walled microspheres into polymer nerve guides for the sustained delivery of glial cell line-derived neurotrophic factor. Biomaterials. 2010;31:2313-22. doi: 10 1016/j.biomaterials.2009.11.075.

16. Crigler L, Robey RC, Asawachaicharn A, Gaupp D, Phinney DG. Human mesenchymal stem cell subpopulations express a variety of neuroregulatory molecules and promote neuronal cell survival and neuritogenesis. Exp Neurol. 2006;198:54-64. doi:10.1016/j.expneurol. 2005.10.029.

17. Guo S, Lo EH. Dysfunctional cell-cell signaling in the neurovascular unit as a paradigm for central nervous system disease. Stroke. 2009;40:54-7. doi:10. 1161/STROKEAHA.108.534388.

18. Mahay D, Terenghi G, Shawcross SG. Growth factors in mesenchymal stem cells following glial-cell differentiation. Biotechnol Appl Biochem. 2008;51: 167-76. doi:10.1042/BA20070212.

19. Mahay D, Terenghi G, Shawcross SG. Schwann cell mediated trophic effects by differentiated mesenchymal stem cells. Exp Cell Res. 2008;314:2692-701. doi:10.1016/j.yexcr.2008.05.013.
20. Ranganath SH, Levy O, Inamdar MS, Karp JM. Harnessing the mesenchymal stem cell secretome for the treatment of cardiovascular disease. Cell Stem Cell. 2012;10:244-58. doi:10.1016/j.stem.2012.02.005.

21. Oliveira JT, Almeida FM, Biancalana A, Baptista AF, Tomaz MA, Melo PA et al. Mesenchymal stem cells in a polycaprolactone conduit enhance median-nerve regeneration, prevent decrease of creatine phosphokinase levels in muscle, and improve functional recovery in mice. Neuroscience. 2010;170:1295-303. doi:10.1016/j.neuroscience.2010.08.042.

22. Ankrum JA, Ong JF, Karp JM. Mesenchymal stem cells: immune evasive, not immune privileged. Nat Biotechnol. 2014;32:252-60. doi:10.1038/nbt.2816.

23. Lavasani M, Thompson SD, Pollett JB, Usas A, Lu A, Stolz DB, et al. Human muscle-derived stem/progenitor cells promote functional murine peripheral nerve regeneration. J Clin Invest. 2014;124. doi:10.1172/JCI44071.eration

24. Chen WCW, Park TS, Murray IR, Zimmerlin L, Lazzari L, Huard J, et al. Cellular kinetics of perivascular MSC precursors. Stem Cells Int. 2013;2013:983059. doi:10.1155/2013/983059

25. Nesti LJ, Jackson WM, Shanti RM, Koehler SM, Aragon AB, Bailey JR, et al. Differentiation potential of multipotent progenitor cells derived from wartraumatized muscle tissue. J Bone Jt Surg Am. 2008;90:2390-8. doi:10.2106/ jbjs.h.00049.

26. Lavasani M, Lu A, Thompson SD, Robbins PD, Huard J, Niedernhofer LJ. Isolation of muscle-derived stem/progenitor cells based on adhesion characteristics to collagen-coated surfaces. Methods Mol. Biol. 2013;976:53-65. doi:10.1007/978-1-62703-317-6_5.

27. Bulken-Hoover JD, Jackson WM, Ji Y, Volger JA, Tuan RS, Nesti LJ. Inducible expression of neurotrophic factors by mesenchymal progenitor cells derived from traumatically injured human muscle. Mol Biotechnol. 2012;51:128-36. doi:10.1007/s12033-011-9445-z

28. Jackson WM, Aragon AB, Djouad F, Song Y, Koehler SM, Nesti $\sqcup$, et al. Mesenchymal progenitor cells derived from traumatized human muscle. J Tissue Eng Regen Med. 2009:3:129-38. doi:10.1002/term.149.

29. Jackson WM, Alexander PG, Bulken-Hoover JD, Vogler JA, Ji Y, McKay P, et al. Mesenchymal progenitor cells derived from traumatized muscle enhance neurite growth. J Tissue Eng Regen Med. 2013;7:443-51. doi:10.1002/term.539.

30. Lozito TP, Jackson WM, Nesti LJ, Tuan RS. Human mesenchymal stem cells generate a distinct pericellular zone of MMP activities via binding of MMPs and secretion of high levels of TIMPs. Matrix Biol. 2014;34:132-43. doi:10.1016/j.matbio.2013.10.003.

31. Kolf CM, Cho E, Tuan RS. Mesenchymal stromal cells. Biology of adult mesenchymal stem cells: regulation of niche, self-renewal and differentiation. Arthritis Res Ther. 2007;9:204. doi:10.1186/ar2116.

32. Lozito TP, Taboas JM, Kuo CK, Tuan RS. Mesenchymal stem cell modification of endothelial matrix regulates their vascular differentiation. J Cell Biochem. 2009;107:706-13. doi:10.1002/jcb.22166.

33. Lozito TP, Kuo CK, Taboas JM, Tuan RS. Human mesenchymal stem cells express vascular cell phenotypes upon interaction with endothelial cell matrix. J Cell Biochem. 2009;107:714-22. doi:10.1002/jcb.22167.

34. Lundborg G. Nerve injury and repair. New York: Churchill Livingstone; 1988.

35. Chauvet S, Burk K, Mann F. Navigation rules for vessels and neurons: cooperative signaling between VEGF and neural guidance cues. Cell Mol Life Sci. 2013:70:1685-703. doi:10.1007/s00018-013-1278-4.

36. Hansen T, Moss A, Brindle N. Vascular endothelial growth factor and angiopoietins in neurovascular regeneration and protection following stroke. Curr Neurovasc Res. 2008;5:236-45. doi:10.2174/ 156720208786413433

37. Sondell M, Sundler F, Kanje M. Vascular endothelial growth factor is a neurotrophic factor which stimulates axonal outgrowth through the flk-1 receptor. Neuroscience. 2000;12:4243-54.

38. Pan Z, Fukuoka S, Karagianni N, Guaiquil VH, Rosenblatt MI. Vascular endothelial growth factor promotes anatomical and functional recovery of injured peripheral nerves in the avascular cornea. FASEB J. 2013; 1-12. doi:10.1096/fj.12-225185

39. Bean AC, Tuan RS. Fiber diameter and seeding density influence chondrogenic differentiation of mesenchymal stem cells seeded on electrospun poly( $\varepsilon$-caprolactone) scaffolds. Biomed Mater. 2015;10:15018. doi:10.1088/1748-6041/10/1/015018.

40. Doube M, Kłosowski MM, Arganda-Carreras I, Cordelières FP, Dougherty RP, Jackson JS, et al. BoneJ: free and extensible bone image analysis in Image. J Bone. 2010;47:1076-9. doi:10.1016/j.bone.2010.08.023. 
41. Liu ZQ. Scale space approach to directional analysis of images. Appl Opt. 1991;30:1369-73. doi:10.1364/AO.30.001369.

42. Dezawa M, Ishikawa H, Itokazu Y, Yoshihara T, Hoshino M, Takeda S, et al. Bone marrow stromal cells generate muscle cells and repair muscle degeneration. Science. 2005;309:314-7. doi:10.1126/science.1110364.

43. Schindelin J, Arganda-Carreras I, Frise E, Kaynig V, Longair M, Pietzsch T, et al. Fiji: an open-source platform for biological-image analysis. Nat Methods. 2012;9:676-82. doi:10.1038/nmeth.2019.

44. Gundersen RW. Response of sensory neurites and growth cones to patterned substrata of laminin and fibronectin in vitro. Dev Biol. 1987;121:423-31.

45. Kato G, Wakabayashi K. Effect of polylysine-bound laminin on human retinoblastoma cell lines. In Vitro Cell Dev Biol. 1988;24:274-80.

46. Preibisch S, Saalfeld S, Tomancak P. Globally optimal stitching of tiled 3D microscopic image acquisitions. Bioinformatics. 2009;25:1463. doi:10.1093/ bioinformatics/btp184.

47. Reneker DH, Yarin AL. Electrospinning jets and polymer nanofibers. Polymer. 2008;49:2387-425. doi: 10.1016/j.polymer.2008.02.002.

48. Miyamoto M, Murphy T, Schnaar R, Coyle J. Antioxidants protect against glutamate-induced cytotoxicity in a neuronal cell line. J Pharmacol Exp Ther. 1989;250:1132-40.

49. Cameron NE, Cotter MA, Maxfield EK. Anti-oxidant treatment prevents the development of peripheral nerve dysfunction in streptozotocin-diabetic rats. Diabetologia. 1993;36:299-304. doi:10.1007/BF00400231.

50. Pinholt C, Hartvig RA, Medlicott NJ, Jorgensen L. The importance of interfaces in protein drug delivery - why is protein adsorption of interest in pharmaceutical formulations? Expert Opin Drug Deliv. 2011;8:949-64. doi:10.1517/17425247.2011.577062.

51. Jin K, Zhu Y, Sun Y, Mao XO, Xie L, Greenberg DA. Vascular endothelial growth factor (VEGF) stimulates neurogenesis in vitro and in vivo. Proc Natl Acad Sci U S A. 2002;99:11946-50. doi:10.1073/pnas.182296499.

52. Woodard GE, Ji Y, Christopherson GT, Wolcott KM, Hall DJ, Jackson WM, et al. Characterization of discrete subpopulations of progenitor cells in traumatic human extremity wounds. PLoS One. 2014;9:e114318. doi:10.1371/journal.pone.0114318.

53. Tamaki T, Hirata M, Soeda S, Nakajima N, Saito K, Nakazato K, et al. Preferential and comprehensive reconstitution of severely damaged sciatic nerve using murine skeletal muscle-derived multipotent stem cells. PLoS One. 2014;9:e91257. doi:10.1371/journal.pone.0091257.

54. Tamaki T. Bridging long gap peripheral nerve injury using skeletal musclederived multipotent stem cells. Neural Regen Res. 2014;9:1333-6. doi:10.4103/1673-5374.137582.

55. Jackson WM, Lozito TP, Djouad F, Kuhn NZ, Nesti L, Tuan RS. Differentiation and regeneration potential of mesenchymal progenitor cells derived from traumatized muscle tissue. J Cell Mol Med. 2011;15:2377-88. doi:10.1111/j.1582-4934.2010.01225.x.

56. Storm T, Wulf K, Teske M, Löbler M, Kundt G, Luderer F, et al. Chemical activation and changes in surface morphology of poly( $\varepsilon$-caprolactone) modulate VEGF responsiveness of human endothelial cells. J Mater Sci Mater Med. 2014;25:2003-15. doi:10.1007/s10856-014-5226-0.

57. Kingham PJ, Kolar MK, Novikova LN, Novikov LN, Wiberg M. Stimulating the neurotrophic and angiogenic properties of human adipose-derived stem cells enhances nerve repair. Stem Cells Dev. 2014;23:741-54. doi:10.1089/scd.2013.0396.

58. Brohlin M, Kingham PJ, Novikova LN, Novikov LN, Wiberg M. Aging effect on neurotrophic activity of human mesenchymal stem cells. PLoS One. 2012;7:67-75. doi:10.1371/journal.pone.0045052.

59. Paradisi M, Alviano F, Pirondi S, Lanzoni G, Fernandez M, Lizzoi G, et al. Human mesenchymal stem cells produce bioactive neurotrophic factors: source, individual variability and differentiation issues. Int J Immunopathol Pharmacol. 2014:27:391-402.

\section{Submit your next manuscript to BioMed Central and we will help you at every step:}

- We accept pre-submission inquiries

- Our selector tool helps you to find the most relevant journal

- We provide round the clock customer support

- Convenient online submission

- Thorough peer review

- Inclusion in PubMed and all major indexing services

- Maximum visibility for your research

Submit your manuscript at www.biomedcentral.com/submit
Biomed Central 